Proyecciones Journal of Mathematics

Vol. 28, N ${ }^{o}$ 2, pp. 155-167, August 2009.

Universidad Católica del Norte

Antofagasta - Chile

\title{
ON THE LOCAL CONVERGENCE OF A MIDPOINT METHOD IN BANACH SPACES UNDER A GAMMA-TYPE CONDITION
}

\author{
IOANNIS K. ARGYROS \\ CAMERON UNIVERSITY, U. S. A. \\ Received: October 2008. Accepted : June 2009
}

\begin{abstract}
In this study we are concerned with the problem of approximating a locally unique solution of an operator equation in a Banach space setting using the midpoint method, introduced by us in [5], [6]. Here, we use gamma-type condition to provide a local convergence analysis. Our results compare favorably with the relevant ones in [9], [11], [12][14]. In particular our radius of convergence is larger. Numerical examples are also provided.
\end{abstract}

AMS (MOS) Subject Classification codes : 65G99, 65K10, 47H17, 49M15.

Key Words : Midpoint method, Banach space, Gamma-type condition, radius of convergence, local convergence, Fréchet-derivative. 


\section{Introduction}

In this study we are concerned with the problem of approximating a locally unique solution $x^{*}$ of the nonlinear equation

$$
F(x)=0,
$$

where $F$ is a thrice-Fréchet-differentiable operator defined on a convex subset $D$ of a Banach space $X$ with values in a Banach space $Y$.

We revisit the midpoint method given for $x_{0} \in D$ by $\mathrm{y}_{n}=x_{n}-$ $F^{\prime}\left(x_{n}\right)^{-1} F\left(x_{n}\right)$,

$\left.x_{n+1}=x_{n}-F^{\prime}\left(z_{n}\right)^{-1} F\left(x_{n}\right), \quad z_{n}=\frac{x_{n}+y_{n}}{2}, n \geq 0\right)$, to generate a sequence $\left\{x_{n}\right\}(n \geq 0)$ approximating $x^{*}[5],[6]$.

Let us illustrate how this method is conceived:

We start with the identity

$$
F(x)=F(y)=\int_{0}^{1} F^{\prime}(y+t(x-y)) d t(x-y), \text { for all } x, y \in D .
$$

If $x^{*}$ is a solution of equation (1.1), then identity (1.3) gives

$$
F(x)=\int_{0}^{1} F^{\prime}\left(x+t\left(x^{*}-x\right)\right) d t\left(x^{*}-x\right) .
$$

The linear operator in (1.4) can be approximated in different ways [3], [4], [13].

If for example

$$
\int_{0}^{1} F^{\prime}\left(x+t\left(x^{*}-x\right)\right) d t \doteq F^{\prime}(x), \text { for all } x \in D,
$$

then (1.4) suggests the famous Newton's method 1-[14]:

$$
x_{n+1}=x_{n}-F^{\prime}\left(x_{n}\right)^{-1} F\left(x_{n}\right) \quad(n \geq 0) .
$$

Another choice is given by

$$
\int_{0}^{1} F^{\prime}\left(x+t\left(x^{*}-x\right)\right) d t=F^{\prime}\left(\frac{x^{*}+x}{2}\right), \text { for all } x \in D,
$$

which leads to the implicit iteration:

$$
x_{n+1}=x_{n}-F^{\prime}\left(\frac{x_{n}+x_{n+1}}{2}\right)^{-1} F\left(x_{n}\right), \quad(n \geq 0) .
$$


Unfortunately iterates in (1.8) can only be commuted in very restrictive cases. That is why we consider $y_{n}$ given in (1.2) as a suitable replacement for iterate $x_{n+1}(n \geq 0)$. Hence, we arrive at the midpoint method (1.2). This method was first introduced by us in [5], [6], where the cubically semilocal convergence was established. Recently in the elegant works by $\mathrm{H}$. H. Homeier [9], and A. Y. Özban [11] the local convergence of the midpoint (1.2) was studied only in the real or complex space.

They also showed the cubical convergence of the midpoint method (1.2) by basically assuming that $x^{*}$ is a simple zero and $F$ is sufficiently many times differentiable (more than 2).

Here we provide our local convergence analysis but on a Banach space setting and using hypotheses reaching only until the third Fréchet-derivative. We also show that the midpoint method is of order three, and also provide upper error estimates on the distances

$$
\left\|x_{n+1}-x^{*}\right\| \text { and }\left\|y_{n}-x^{*}\right\|(n \geq 0) .
$$

In order for us to provide the local convergence analysis we use the concept of a $\gamma$-type condition introduced for the semilocal convergence condition in the elegant works by Wang (see e.g. [14]. Here we show that although we are using a more general concept of the $\gamma$-condition than in [14] (see, $(2.3),(2.4),(2.22),(2.23)$ and (2.27) we can obtain a larger radius of convergence. The same favorable comparison is made (see (2.26) between the radius of convergence given by Rheinboldt in [12] (for Newton's method, using the stronger domain based instead of point information used here).

Finally, numerical examples are also provided.

\section{Local convergence analysis of the midpoint method (1.2}

Let us define scalar function $f$ on $\left[0, \frac{1}{\gamma}\right)$ by

$$
f(t)=\beta-t+\frac{\gamma t^{2}}{1-\gamma t},
$$

where $\beta \geq 0$, and $\gamma>0$ are given. It is known [14] that if

$$
\alpha=\beta \gamma \leq 3-2 \sqrt{2},
$$

then function $f$ has two roots

$$
t^{*}=\frac{1+\alpha-\sqrt{(1+\alpha)^{2}-8 \alpha}}{2 \gamma}, t^{* *}=\frac{1+\alpha+\sqrt{(1+\alpha)^{2}-8 \alpha}}{2 \gamma}
$$


satisfying

$$
\beta \leq t^{*} \leq\left(1+\frac{1}{\sqrt{2}}\right) \beta \leq\left(1-\frac{1}{\sqrt{2}}\right) \frac{1}{\gamma} \leq t^{* *} \leq \frac{1}{2 \gamma}
$$

We shall assume throughout this paper the $\gamma$-conditions:

there exists a zero $x^{*} \in D$ of operator $F$ such that

$$
F^{\prime}\left(x^{*}\right)^{-1} \in L(Y, X)
$$

Operator $F$ is thrice Fréchet-differentiable on $D$, and for all $x \in D$

$$
\left\|F^{\prime}\left(x^{*}\right)^{-1} F^{\prime \prime}\left(x^{*}\right)\right\| \leq 2 \gamma,
$$

and

$$
\left\|F^{\prime}\left(x^{*}\right)^{-1} F^{\prime \prime \prime}(x)\right\| \leq \frac{6 \gamma^{2}}{\left(1-\gamma\left\|x-x^{*}\right\|\right)^{4}}=f^{\prime \prime \prime}\left(\left\|x-x^{*}\right\|\right) .
$$

In view of (2.1), we have

$$
\begin{gathered}
f^{\prime}(t)=\frac{1-2(1-\gamma t)^{2}}{(1-\gamma r)^{2}} \\
f^{\prime \prime}(t)=\frac{2 \gamma}{(1-\gamma t)^{3}},
\end{gathered}
$$

and

$$
f^{\prime \prime \prime}(t)=\frac{6 \gamma^{2}}{(1-\gamma t)^{4}}
$$

We need the following Lemma:

Lemma 1. Under the $\gamma$-conditions, and for all $x \in U\left(x^{*},\left(1-\frac{1}{\sqrt{2}}\right) \frac{1}{\gamma}=r_{0}\right)$

$=\left\{x \in X:\left\|x-x^{*}\right\|<r_{0}\right\} \subseteq D$ the following estimates hold:

$\left\|F^{\prime}\left(x^{*}\right)^{-1} F^{\prime \prime}(x)\right\| \leq f^{\prime \prime}\left(\left\|x-x^{*}\right\|\right)$,

$F^{\prime}(x)^{-1} \in L(Y, X)$,

and

$$
\left\|F^{\prime}(x)^{-1} F^{\prime}\left(x^{*}\right)\right\| \leq-\frac{1}{f^{\prime}\left(\left\|x-x^{*}\right\|\right)} .
$$


Proof. Using the $\gamma$-condition, and the properties of function $f$, we obtain in turn: $\left\|F^{\prime}\left(x^{*}\right)^{-1} F^{\prime \prime}(x)\right\| \leq$

$\leq\left\|F^{\prime}\left(x^{*}\right)^{-1} F^{\prime \prime}\left(x^{*}\right)\right\|$

$+\left\|F^{\prime}\left(x^{*}\right)^{-1}\left[F^{\prime \prime}(x)-F^{\prime \prime}\left(x^{*}\right)\right]\right\|$

$=\left\|F^{\prime}\left(x^{*}\right)^{-1} F^{\prime \prime}\left(x^{*}\right)\right\|+\left\|\int_{0}^{1} F^{\prime}\left(x^{*}\right)^{-1} F^{\prime \prime}\left(x^{*}+t\left(x-x^{*}\right)\right)\left(x-x^{*}\right) d t\right\|$

$\leq 2 \gamma+\int_{0}^{1} f^{\prime \prime}\left(t\left\|x-x^{*}\right\|\right)\left\|x-x^{*}\right\| d t$

$=2 \gamma+f^{\prime \prime}\left(\left\|x-x^{*}\right\|\right)-f^{\prime \prime}(0)=f^{\prime \prime}\left(\left\|x-x^{*}\right\|\right)$.

Moreover, we have $\left\|F^{\prime}\left(x^{*}\right)^{-1}\left[F^{\prime}(x)-F^{\prime}\left(x^{*}\right)\right]\right\|=$

$=\left\|F^{\prime}\left(x^{*}\right)^{-1} \int_{0}^{1} F^{\prime \prime}\left(x^{*}+t\left(x-x^{*}\right)\right)\left(x-x^{*}\right) d t\right\|$

$\leq \int_{0}^{1} f^{\prime \prime}\left(t\left\|x-x^{*}\right\|\right)\left\|x-x^{*}\right\| d t$

$=f^{\prime}\left(\left\|x-x^{*}\right\|\right)-f^{\prime}(0)=f^{\prime}\left(\left\|x-x^{*}\right\|\right)+1<1$.

It follows by the Banach Lemma on invertible operators [4], [10] that

$$
F^{\prime}(x)^{-1} \in L(Y, X)
$$

and

$$
\begin{aligned}
& \left\|F^{\prime}(x)^{-1} F^{\prime}\left(x^{*}\right)\right\| \leq \frac{1}{1-\left\|F^{\prime}\left(x^{*}\right)^{-1}\left[F^{\prime}(x)-F^{\prime}\left(x^{*}\right)\right]\right\|} \\
& \leq-\frac{1}{f^{\prime}\left(\left\|x-x^{*}\right\|\right)} . \\
& \text { That completes the proof of the Lemma. }
\end{aligned}
$$

It is convenient for us to define:

sequences $\left\{a_{n}\right\},\left\{b_{n}\right\},\left\{\bar{c}_{n}\right\},\left\{c_{n}\right\}$ and $\left\{d_{n}\right\}$ by

$$
\begin{gathered}
a_{n}=\frac{\gamma}{1-\gamma\left\|x_{n}-x^{*}\right\|}, \\
b_{n}=\frac{\gamma^{2}}{4\left(1-\gamma\left\|x_{n}-x^{*}\right\|\right)}, \\
c_{n}=\frac{\left(1-\gamma\left\|x_{n}-x^{*}\right\|\right)^{2}}{2\left(1-\gamma\left\|x_{n}-x^{*}\right\|\right)^{2}-1} \\
\bar{c}_{n}=\frac{\left(1-\gamma\left\|z_{n}-x^{*}\right\|\right)^{2}}{2\left(1-\gamma\left\|z_{n}-x^{*}\right\|\right)^{2}-1},
\end{gathered}
$$

and

$$
d_{n}=\frac{\gamma}{4\left(1-\frac{\gamma}{2}\left\|x_{n}-x^{*}\right\|\right)\left[1-\frac{\gamma}{2}\left(\left\|x_{n}-x^{*}\right\|+\left\|y_{n}-x^{*}\right\|\right)\right]} ;
$$

functions $a, b, \bar{c}, c, d$ on $\left[0, r_{0}\right)$ by 


$$
a(r)=\frac{r}{1-r}, b(r)=\frac{r^{2}}{4(1-r)}, \bar{c}(r)=c(r)=\frac{(1-r)^{2}}{2(1-r)^{2}-1}
$$

and

$$
d(r)=\frac{r}{4\left(1-\frac{r}{2}\right)(1-r)} .
$$

It is simple algebra to see that system of inequalities

$$
a(r) c(r) \leq 1
$$

$$
c(r)[b(r)+d(r)] \leq 1
$$

is satisfied for all

$$
r \in\left[0, \frac{5-\sqrt{13}}{6}\right) .
$$

We shall also use the identities [4]: $\mathrm{F}\left(\mathrm{x}^{*}\right)-F(x)-F^{\prime}(x)\left(x^{*}-x\right)=$ $=\int_{0}^{1} F^{\prime \prime}\left[x+t\left(x^{*}-x\right)\right](1-t)\left(x^{*}-x\right)^{2} d t$,

$\mathrm{F}(\mathrm{x})-\mathrm{F}(\mathrm{y})-\mathrm{F}^{\prime}(z)(x-y)$

$=\frac{1}{4} \int_{0}^{1}(1-t)\left[F^{\prime \prime}\left(z+\frac{t}{2}(x-y)\right)-F^{\prime \prime}\left(z+\frac{t}{2}(y-x)\right)\right](x-y)(x-y) d t$ $=\frac{1}{4} \int_{0}^{1} F^{\prime \prime \prime}\left[z+\frac{t}{2}(y-x)+s t(x-y)\right] t(1-t)(x-y)^{3} d s d t$, and

$$
F^{\prime}(z)-F^{\prime}\left(\frac{x+x^{*}}{2}\right)=\int_{0}^{1} F^{\prime \prime}\left[\frac{x+x^{*}}{2}+t\left(\frac{y-x^{*}}{2}\right)\right]\left(\frac{y-x^{*}}{2}\right) d t
$$

for $z=\frac{x+y}{2}$, and all $x, y \in D$.

We can show the local convergence theorem for the midpoint method (1.2):

Theorem 2. Under the $\gamma$-condition for $\bar{U}\left(x^{*}, r^{*}=\frac{5-\sqrt{13}}{6 \gamma}\right) \subseteq D$, sequences $\left\{x_{n}\right\},\left\{y_{n}\right\}$ generated by the midpoint method (1.2) are well defined, remain in $U\left(x^{*}, r^{*}\right)$ for all $n \geq 0$, and converge to the unique solution $x^{*}$ of equation (1.1) in $\bar{U}\left(x^{*}, r^{*}\right)$ provided that $x_{0} \in U\left(x^{*}, r^{*}\right)$.

Moreover the following estimates hold for all $n \geq 0$ :

$$
\left\|y_{n}-x^{*}\right\| \leq c_{n} a_{n}\left\|x_{n}-x^{*}\right\|^{2},
$$

and

$$
\begin{aligned}
& \quad\left\|x_{n+1}-x^{*}\right\| \leq \bar{c}_{n}\left[b_{n}\left\|x_{n}-x^{*}\right\|^{2}+d_{n}\left\|y_{n}-x^{*}\right\|\right]\left\|x_{n}-x^{*}\right\| \\
& \leq \bar{c}_{n}\left[b_{n}+d_{n} c_{n} a_{n}\right]\left\|x_{n}-x^{*}\right\|^{3} .
\end{aligned}
$$


Proof. By Lemma $1, F^{\prime}\left(x_{0}\right)^{-1} \in L(Y, X)$, since $x_{0} \in U\left(x^{*}, r^{*}\right) \subseteq D$. Therefore $y_{0}$ given by (1.2) for $n=0$ is well defined. We shall show that $y_{0} \in U\left(x^{*}, r^{*}\right)$, and (2.11) holds for $n=0$.

Using (1.2), (2.5), (2.6), (2.7) and (2.9) we obtain $\left\|y_{0}-x^{*}\right\|=$ $=\|\left[F^{\prime}\left(x_{0}\right)^{-1} F^{\prime}\left(x^{*}\right)\right]\left[F^{\prime}\left(x^{*}\right)^{-1} \int_{0}^{1} F^{\prime \prime}\left[x^{*}+t\left(x_{0}-x^{*}\right)\right](1-t)\left(x_{0}-x^{*}\right)^{2} d t \|\right.$ $\leq\left\|F^{\prime}\left(x_{0}\right)^{-1} F\left(x^{*}\right)\right\| \cdot\left\|F^{\prime}\left(x^{*}\right)^{-1} \int_{0}^{1} F^{\prime \prime}\left[x_{0}+t\left(x^{*}-x_{0}\right)\right](1-t)\left(x^{*}-x_{0}\right)^{2} d t\right\|$ $\leq c_{0} \int_{0}^{1} f^{\prime \prime}\left[(1-t)\left\|x^{*}-x_{0}\right\|\right](1-t)\left\|x^{*}-x_{0}\right\|^{2} d t$ $\leq c_{0} a_{0}\left\|x^{*}-x_{0}\right\|^{2} \leq c(r) a(r)\left\|x^{*}-x_{0}\right\| \leq\left\|x^{*}-x_{0}\right\| \leq r^{*}\left(\right.$ for $\left.r=\gamma\left\|x^{*}-x_{0}\right\|\right)$,

which imply $y_{0} \in U\left(x^{*}, r^{*}\right)$, and (2.13) holds for $n=0$. Let us assume $x_{k}, y_{k-1} \in U\left(x^{*}, r^{*}\right)$, and $(2.13)$ holds for $k=0,1, \ldots, n$. It then follows for $y_{k}, x_{k}$ replacing $y_{0}, x_{0}$ in $(2.15)$ that $y_{k} \in U\left(x^{*}, r^{*}\right)$ and (2.13) holds for $n=k$.

We shall show $x_{k+1} \in U\left(x^{*}, r^{*}\right)$, and (2.14) holds.

In view of the identity $\mathrm{x}_{k+1}-x^{*}=x_{k}-x^{*}-F^{\prime}\left(z_{k}\right)^{-1} F\left(x_{k}\right)$

$=F^{\prime}\left(z_{k}\right)^{-1}\left[F^{\prime}\left(z_{k}\right)\left(x_{k}-x^{*}\right)-F\left(x_{k}\right)+F\left(x^{*}\right)\right]$

$=-F^{\prime}\left(z_{k}\right)^{-1}\left[F\left(x^{*}\right)-F\left(x_{k}\right)-F^{\prime}\left(\frac{x_{k}+x^{*}}{2}\right)\left(x^{*}-x_{k}\right)\right]$

$+F^{\prime}\left(z_{k}\right)^{-1}\left[F^{\prime}\left(z_{k}\right)-F^{\prime}\left(\frac{x_{k}+x^{*}}{2}\right)\right]\left(x^{*}-x_{k}\right)$,

(2.4), (2.5), (2.6), (2.8), (2.9), (2.10) and (2.12) we have in turn

$\left\|x_{k+1}-x^{*}\right\| \leq$

$\leq \bar{c}_{k}\left\{\frac{1}{4} \| \int_{0}^{1} F^{\prime}\left(x^{*}\right)^{-1} F^{\prime \prime \prime}\left[\frac{x_{k}+x^{*}}{2}+\frac{t}{2}\left(x_{k}-x^{*}\right)+s t\left(x^{*}-x_{k}\right)\right]\right.$

$t(1-t)\left(x^{*}-x_{k}\right)^{3} d s d t \|+$

$\left.+\left\|\int_{0}^{1} F^{\prime}\left(x^{*}\right)^{-1} F^{\prime \prime}\left[\frac{x_{k}+x^{*}}{2}+t\left(\frac{y_{k}-x^{*}}{2}\right)\right]\left(\frac{y_{k}-x^{*}}{2}\right) d t\left(x_{k}-x^{*}\right)\right\|\right\}$

$\leq \bar{c}_{k}\left[b_{k}\left\|x_{k}-x^{*}\right\|^{2}+d_{k}\left\|y_{k}-x^{*}\right\|\right]\left\|x_{k}-x^{*}\right\|$

$\leq c(r)[b(r)+d(r)]\left\|x_{k}-x^{*}\right\|<\left\|x_{k}-x^{*}\right\| \leq r^{*}$

which imply $x_{k+1} \in U\left(x^{*}, r^{*}\right)$ and (2.14) holds for $n=k$.

That completes the induction.

It also follows from (2.15) (for $x_{0}, y_{0}$ replaced by $\left.x_{k}, y_{k}\right)$, and (2.17) that $\lim _{k \rightarrow \infty} y_{k}=x^{*}=\lim _{k \rightarrow \infty} x_{k}$,

Finally, to show uniqueness let $y^{*} \in \bar{U}\left(x^{*}, r^{*}\right)$ be a solution of equation (1.1).

Using the identity

$$
F\left(x^{*}\right)-F\left(y^{*}\right)=L\left(x^{*}-y^{*}\right),
$$

where, 


$$
L=\int_{0}^{1} F^{\prime}\left(y^{*}+t\left(x^{*}-y^{*}\right)\right) d t
$$

and Lemma 1 for $x$ replaced by $y^{*}+t\left(x^{*}-y^{*}\right)$ that $L^{-1}$ exists. Hence, by (2.19), we deduce $x^{*}=y^{*}$.

That completes the proof of the theorem.

Remark 3. As noted in [4], [7], [13] the local results obtained here can be used for projection method such as Arnoldi's, the generalized minimum residual method (GMRES), the generalized conjugate residual method (GCR), for combined Newton/finite projection methods, and in connection with the mesh independence principle to develop the cheapest and most efficient mesh refinement strategies.

Remark 4. The local results can also be used to solve equations of the form $F(x)=0$, where $F^{\prime}$ satisfies the autonomous differential equation [4]

$$
F^{\prime}(x)=P(F(x))
$$

where $P: Y \rightarrow X$ is a known continuous operator. Since $F^{\prime}\left(x^{*}\right)=$ $P\left(F\left(x^{*}\right)\right)=P(0)$, we can apply our results without actually knowing the solution $x^{*}$ of equation (1.1).

Example 5. Let $X=Y=\mathbf{R}, D=U(0,1)$, and define function $F$ on $D$ by

$$
F(x)=e^{x}-1 .
$$

Then, note that we can set $P(x)=x+1$ in (2.20). We must have that conditions (2.3) and (2.4) hold for some $\gamma \geq 0$. It can easily be seen that we can set $\gamma=\frac{1}{2}$. Hence the radius of convergence is $r^{*}=r_{A}=2\left(\frac{5-\sqrt{13}}{6}\right)=$ .464816242 .

The radius of convergence $r_{w}$ in [14] is given by

$$
r_{w}=\frac{1}{2 \gamma^{*}}(3-2 \sqrt{2})
$$

with

$$
\gamma^{*}=\sup _{k \geq 2}\left\|F^{\prime}\left(x^{*}\right)^{-1} F^{(k)}\left(x^{*}\right)\right\|^{\frac{1}{k-1}} \leq \frac{1}{2}
$$


Therefore, (2.22) gives

$$
r_{w} \leq 3-2 \sqrt{2}=\cdot 171573 .
$$

Moreover, Rheinboldt ball [12] $r_{R}$ is given by

$$
r_{R}=\frac{2}{3 \ell},
$$

where $\ell$ is the Lipschitz constant in condition:

$$
\left\|F\left(x^{*}\right)^{-1}\left[F^{\prime}(x)-F^{\prime}(y)\right]\right\| \leq \ell\|x-y\|
$$

for all $x, y \in D$.

Using (2.21) and (2.25) we get $\ell=e$.

That is

$$
r_{R}=\cdot 245252961
$$

Hence, we deduce

$$
r_{w}<r_{R}<r_{A}
$$

By comparing $r_{A}$ and $r_{w}$ we see that it is always true that

$$
r_{w}<r_{A}
$$

Moreover note that under (2.2) the existence of $x^{*}$ in $U\left(x_{0}, \frac{1}{\gamma}\left(1-\frac{1}{\sqrt{2}}\right)\right)$ is guaranteed [14]. However, in practice the existence of $x^{*}$ may have been established by another way that avoids condition (2.2). Finally note that enlarging the radius of convergence is very important in computational mathematics since in this case we can obtain a wider range of initial guesses $x_{0}$.

Remark 6. In order for us to compare Midpoint method (1.2) with related methods already in the literature, consider the two-step Newton method [4], [10]:

$$
\begin{aligned}
& y_{n}=x_{n}-F^{\prime}\left(x_{n}\right)^{-1} F\left(x_{n}\right) \quad(n \geq 0) \\
& x_{n+1}=y_{n}-F^{\prime}\left(x_{n}\right)^{-1} F\left(y_{n}\right) \quad(n \geq 0) .
\end{aligned}
$$

Note that Newton's method (1.6) use one function evaluation and one inverse at each step and is of order two.

Midpoint method (1.2) uses one function evaluation and two inverses at each step, but it is of order three. Finally, two step method (2.28) uses one 
inverse and two functions evaluations at each step, and it is also of order three.

In the numerical example that follows, we show that Midpoint method (1.2) can be the fastest of the three.

Example 7. Let $X=Y=\mathbf{R}^{2}$, and define operator $F: \mathbf{R}^{2} \longrightarrow \mathbf{R}^{2}$ by

$$
F\left(x_{1}, x_{2}\right)=\left[\begin{array}{l}
F_{1}\left(x_{1}, x_{2}\right) \\
F_{2}\left(x_{1}, x_{2}\right)
\end{array}\right],
$$

where,

$$
F_{1}\left(x_{1}, x_{2}\right)=2 x_{1}-\frac{1}{9} x_{1}^{2}-x_{2} \quad \text { and } \quad F_{2}\left(x_{1}, x_{2}\right)=-x_{1}+2 x_{2}-\frac{1}{9} x_{2}^{2} .
$$

We use the infinity norm $\|x\|=\|x\|_{\infty}=\max \left(\left\|x_{1}\right\|,\left\|x_{2}\right\|\right)$, and the induced matrix norms. Then, we can easily verify $x^{*}=(9,9)$ is a solution of equation $F(x)=0$.

We also have

$$
\begin{gathered}
F^{\prime}(x)=\left[\begin{array}{cc}
2-\frac{2}{9} x_{1} & -1 \\
-1 & 2-\frac{2}{9} x_{2}
\end{array}\right], \quad F^{\prime \prime}(x)=\left[\begin{array}{cc}
-\frac{2}{9} & 0 \\
0 & -\frac{2}{9}
\end{array}\right] \\
F^{\prime \prime \prime}(x)=\left[\begin{array}{ll}
0 & 0 \\
0 & 0
\end{array}\right]
\end{gathered}
$$

and conditions (2.3) and (2.4) are satisfied for $\gamma=\frac{1}{9}$, and $r^{*}=\frac{5-\sqrt{13}}{6 \gamma}=2.09167308$. We noticed that methods (1.2), (1.6) and (2.28) all converge starting with $x_{0}=(11.4,11.4)$ although this point is outside the convergence ball $\bar{U}\left(x^{*}, r^{*}\right)$. In the table that follows, we compare the three methods. 
Comparison Table

\begin{tabular}{cccc|}
\hline$n$ & $\begin{array}{c}\text { Midpoint Method } \\
(1.2)\end{array}$ & $\begin{array}{c}\text { 2-Step } \\
\text { Newton Method } \\
(2.28)\end{array}$ & $\begin{array}{c}\text { Newton's Method } \\
(1.6)\end{array}$ \\
\hline 1 & {$\left[\begin{array}{l}9.08476821 \\
9.08476821\end{array}\right]$} & {$\left[\begin{array}{l}9.17649271 \\
9.17649271\end{array}\right]$} & {$\left[\begin{array}{l}9.41739131 \\
9.41739131\end{array}\right]$} \\
2 & {$\left[\begin{array}{l}9.00000731 \\
9.00000731\end{array}\right]$} & {$\left[\begin{array}{l}9.00317622 \\
9.00317622\end{array}\right]$} & {$\left[\begin{array}{l}9.01771422 \\
9.01771422\end{array}\right]$} \\
3 & {$\left[\begin{array}{l}9.00000000 \\
9.00000000\end{array}\right]$} & {$\left[\begin{array}{l}9.00001142 \\
9.00001142\end{array}\right]$} & {$\left[\begin{array}{l}9.00003473 \\
9.00003473\end{array}\right]$} \\
4 & & {$\left[\begin{array}{l}9.00000000 \\
9.00000000\end{array}\right]$} & {$\left[\begin{array}{l}9.00000001 \\
9.00000001\end{array}\right]$} \\
5 & & {$\left[\begin{array}{l}9.00000000 \\
9.00000000\end{array}\right]$} \\
\hline
\end{tabular}

Note that in this case the Midpoint method (1.2) is faster that Newton's method (1.6), and two-step Newton method (2.28).

\section{References}

[1] Allgower, F. L., Böhmer, K., Potra, F. A. and Rheinboldt, W.C. A mesh independence principle for operator equations and their discretizations, SIAM J. Numer. Anal. 23, pp. 160-169, (1986).

[2] Amat, S. and Busquier, S. Convergence and numerical analysis of a family of two-step Steffensen's method, Comput. and Math. with appl., 49 , pp. 13-22, (2005).

[3] Argyros, I. K. A unifying local-semilocal convergence analysis and applicastions for two-point Newton-like methods in Banach space, J. Math. Anal. Appl., 298, pp. 374-397; (2004). 
[4] Argyros, I. K. Approximate solution of operator equations with applications, World Scientific Publ. Co. Ptl. Ltd., Hackensack, N. J., U. S. A., (2005).

[5] Argyros, I. K. and Chen, D. On the midpoint methods for solving equations in Banach spaces, Appl. Math. Letter, Vol. 5, No.4, pp. 7-9, (1992).

[6] Argyros, I. K., and Chen, D. On the midpoint iterative method for solving nonlinear operator equations and applications to the solution of integral equations, Revue d'Analyse Numérique et de Théorie de l'Approximation, Tome 23, fasc. 2, pp. 139-152, (1994).

[7] Brown, P. N. A local convergence theory for combined inexactNewton/finite-difference projection methods, SIAM J. Numer. Anal. 24, pp. 407-434, (1987).

[8] Gutierrez, J. M., Hernandez, M. A. and Salanova, M.A. Accessibility of solutions by Newton's method, J. Comput. Math. 57, pp. 239-247, (1995).

[9] Homeir, H. H. H. A modified Newton method for root finding with cubic convergence, J. Comput. Appl. Math., 157, pp. 227-230, (2003).

[10] Kantorovich, L. V., and Akilov, G. P. Functional Analysis in normed spaces, Pergamon Press, Oxford, (1982).

[11] Özban, A. Y. Some new variants of Newton's method, Appl. Math. Letters, 17, pp. 677-682, (2004).

[12] Rheinboldt, W. C. An adaptive continuation process for solving systems of nonlinear equations, Banach Center Publ. 3, pp. 129-142, (1977).

[13] Ypma, T. J. Local convergence of inexact Newton Methods,SIAM J. Numer, Anal., 21, pp. 583-590, (1984).

[14] Zhao, F. and Wang, D. The theory of Smale's point estimation and its applications, J. Comput. Appl. Math., 60, pp. 253-269, (1995). 


\section{Ioannis K. Argyros}

Cameron University

Department of Mathematical Sciences

Lawton, OK 73505

U. S. A.

e-mail : iargyros@cameron.edu 